\title{
SÉANCE DU 23 OCTOBRE 1914
}

\author{
PRÉSIDENGE DE M. P.-A. DANGEARD.
}

M. F. Moreau, vice-secrétaire, donne lecture du procèsverbal de la dernière séance, dont la rédaction est adoptée.

\section{DONS FAITS A LA SOCIÉTÉ}

Battandier (J.-A.), Le milieu agent modificateur des espèces.

Beauverie (J.), Sur le chondriome d'une Urédinée : Le Puccinia Malvacearum.

- Sur le chondriome des Basidiomycètes.

- La muscardine. Le genre Beauveria Vuillemin.

- Étude d'une maladie des Pêchers dans la vallée du Rhône.

Bonaparte (Prince R.), Fougères d'Afrique de l'herbier du Muséum. Bonnier (G.), Flore complète et illustrée en couleurs de France, Suisse et Belgique, fasc. 26.

Bouly de Lesdain, Recherches sur les Lichens des environs de Dunkerque. $1^{\text {or }}$ supplément.

Briosi (G.), Operosita sino allanno 1912 della Stazione botanica crittogamica in Pavia.

- Cenno sopra Francesco Ginanni.

- Sulla presenza dei cordoni endocellulari nei tessuti della vite e di altra dicotiledoni.

Dalmon (H.), Un parc national en forêt de Fontainebleau.

Daveau (J.), Sur deux Ormes nouveaux de la section Micropetala.

Hayata (B.), Icones Plantarum Formosanarum necnon et Contributiones ad Floram Formosanam. III.

Hickel (R.), Graines et plantes des arbres et arbustes indigènes et communément cullivés en France. $1^{\mathrm{re}}$ et $2^{\mathrm{e}}$ parties.

Jahandiez (E.), Les Iles d'Hijères. Histoire. Description, Géologie. Flore. Faune.

Kufferath (H.), Contribution à la Physiologie d'une Protococcacée nouvelle.

Lecomte (H.), Flore générale de l'Indo-Chine, IV 2, V 2. 
- Lauracées de Chine et d'Indo-Chine.

Litardière (R. de), Contribution à l'étude de la flore de Corse.

Maire (René), La structure et la position systématique du Mapea radiata $P$ at.

- Sur la synonymie et les affinités de l'Hygrophorus Marzuolus.

- Notes critiques sur les Champignons récoltés pendant la session de Grenoble-Annecy de la Société Mycologique de France.

- Études mycologiques. Fasc. I.

- Sur quelques Champignons parasites du littoral normand.

- Contribution à l'Étude de la Flore du Djurjura.

- Contribution à l'Étude des Laboulbéniales de l'Afrique du Nord.

- Remarques sur l'indigénat du Sapin en Normandie.

- La structure et la position systématique des Microstroma et Helostroma.

lieyes Prosper (E.), Les carofitas de España.

Reynier (Alfred), L'Honkenya peploides Ehr. dans le Var.

Roadway (L.), Tasmanian Bryophyta. Mosses.

Roux (Claudius), 25 brochures botaniques.

De Toni, In memoria di Paolo Petit.

Trabut (L.), Le Kumquat, Gitrus japonica. Culture dans le Nord de l'A frique.

Vernham (H.-F.), A monograph of the Genus Sabicea.

Bulletin de la Société d'Études scientifiques d'Angers, XLII-XLIII, 1912-1913.

Revue scientifique du Bourbonnais et du Centre de la France, 1914, fasc. 1-2.

Revue scientifique du Limousin, nos 25 5.-257.

Revue horticole. Journal mensuel des travaux de la Société d'Horticulture et de Botanique des Bouches-du-Rhòne, $\mathrm{n}^{\text {os }} \mathbf{7 1 5}-716$.

Bulletin de la Société des Naturalistes-archéologues du nord de Meuse, XXIV-1912.

Bulletin de la Société d'histoire naturelle de l'Afrique du Nord, V, 2-รั.

La Nuova Notarisia, aprile 1914.

Journal of the College of Agriculture. Imperial University of Tokio, IV, 6 .

Memoirs of the Department of Agriculture in 'India, VI, 4. Report of the Agricultural Research Institute and College Pusa, 1912-13.

Mededeelingen van de Afdeeling voor Plantenziekten, 7. Annals of the Missouri Botanical Garden, I, 1. Journal of agricultural Research (Washington), I, 1. 


\section{$2 \mathrm{BHL}$ Biodiversity Heritage Library}

1914. "SÉance Du 23 Octobre 1914." Bulletin de la Société botanique de France 61, 384-385. https://doi.org/10.1080/00378941.1914.10832589.

View This Item Online: https://www.biodiversitylibrary.org/item/8683

DOI: https://doi.org/10.1080/00378941.1914.10832589

Permalink: https://www.biodiversitylibrary.org/partpdf/161091

\section{Holding Institution}

Missouri Botanical Garden, Peter H. Raven Library

\section{Sponsored by}

Missouri Botanical Garden

\section{Copyright \& Reuse}

Copyright Status: Public domain. The BHL considers that this work is no longer under copyright protection.

This document was created from content at the Biodiversity Heritage Library, the world's largest open access digital library for biodiversity literature and archives. Visit BHL at https://www.biodiversitylibrary.org. 